\title{
SURVEY PAPER ON PERPETRATOR IDENTIFICATION AND ACKNOWLEDGEMENT ON ROAD ACCIDENTS
}

\author{
Arpithanath. $\mathbf{M}^{\mathbf{1}}$, Suraiya Khanum $\mathrm{A}^{\mathbf{2}}$, Umaima Rafi ${ }^{3}$, Vinaya Ananthi $\mathbf{R}^{\mathbf{4}}$, Anand Swamy A $\mathbf{S}^{\mathbf{5}}$ \\ ${ }_{1,2,3,4} 8^{\text {th }}$ Sem, Department of Telecommunication, T. John Institute of Technology, Bangalore, Karnataka \\ ${ }^{5}$ Department of ECE/TCE, T. John Institute of Technology, Bangalore, Karnataka.
}

\begin{abstract}
Vehicles safety and safety of the passengers in the vehicle is an important task. Hence, technology has come to an extent that the vehicles can communicate using vehicle ad hoc network with each other. VANET provides wireless communication between the vehicle and to the road side units (RSU). The accidents can be kept in control by having a free of congestion communication between the vehicles in the network using RACO algorithm. The accidents can be avoided even by reducing the vehicles acceleration in the traffic signal using the RF Xbee modules. The speed of the vehicles can also be reduced by reducing the fuel rate of the vehicles as well.
\end{abstract}

Keywords: VANET; RACO; RF Xbee; RSU.

\section{INTRODUCTION}

The count of vehicle on roads is increasing in a very bold way for every passing year as count increases riskier becomes the transportation. Increased rate of traffic leads to jams and accidents as to overcome this problem there is a need of automatic speed controllers. Automation of driving control of two-wheeler is one of the important needs of the hour. VANET's which are derived from MANET's allows the vehicle to communicate with other vehicle that are in a particular range. By making vehicles to communicate with each other risk of accidents can be minimized efficiently.Vehicle control system is very much needed this system reduces the risk of accidents by indicating a pre indication and also by reducing the speed of the vehicles.

In the further coming topics, we will be looking into the Related Works in Section 2, The Issues in the Existing Systems Technique in Section 3, RACO in the Section 3(a) in which the working and Architecture is discussed . RF Tech in the Section of 3(b) in which the Transceivers and the Hardware and Software Designs is discussed, The Future Scope in the Section 4, and the concluding part in the final Section 5.

\section{RELATED WORKS}

Jaya Sehgal et al.[1], has presented an intelligent route detection/identification approad when some mishap happens in a point to point network system of vehicles. Here, they have used the technology of biologically inspired $\mathrm{p} 2 \mathrm{p}$ communication using the pheromones chemical to retrace the path to reach their home (destination). The basic concept taking place is that when ever a mishap occurs, immediately the incident is being informed to the other vehicles such that other mobile devices can vary their route to avoid the congestion.
Debasmita Mukherjee et al. [2], has presented an idea to discover the shortest available path by applying various Ant Colony Optimization Mechanisms. Here, with the help of protocol of routing, the selection of path by calculation is done. The algorithm which is proposed in this is to find the path which is the shortest where the virtual packets is being stimulated from the start point (Source) till the end point (Destination). Here, the route is chosen at random for the 1st time and hence updation of the routing table takes place. Thus, the route which has the large depth of the chemical substrate is chosen by the Ants following.

Gupta D.K et al.[3], has proposed a new and revised technique which is called Recursive ACO (RACo). Here, an additional term called DePTH is into existence were in that term is used to find the extent of Recursion. The 3 usual methods of finding the recursion is that

a) Tracking of pheromone.

b) Updation of pheromone.

c) City selection.

Thus, this paper concludes by telling that this method finds the best answer to the issues caused in combination.

Rezwana Karim [4], has proposed an idea which is best suitable for providing Safety of the vehicles and comfort of the passenger, which obviously gives an addition to the industry of the vehicle production and the services of the operators. Firstly, here a comparative study has been done which includes a detailed study of all the available measures of safety and the issues related to the different systems. There are various issues to be addressed like in the Access of the Wireless Technology, which has the IEEE $802.11 \mathrm{p}$ and the issues related to the spectrum and the dissemination of the messages, issues based on routing and broadcasting, which includes the Vehicular to vehicular dissemination and finally security and privacy. 


\section{ISSUES IN THE EXISTING SYSTEM TECHNIQUES}

A. Recursive Ant Colony Optimization[ RACO]:

The technique of the Optimization of the Ant Colony is that by looking into the behavior of the Ants, Marco Dorigo, in the year of 1992, started developing the algorithm based on it. The existence of the pheromone chemical substance helps these insects to communicate with each other, and also another advantage of this chemical substance is that they can be used for alerting the other insects during any dangerous situations and so on. Thus even for the vehicular systems, a similar technology is used to alert the devices during any of the dangerous situations.

Working of this Technique:

The chemicals are dropped onto the floor and the following insects walk onto the path which has the highest level of the chemical because, when an insect (ant) follows the trail, they leave their chemicals behind onto the same path too and hence the depth or level of the chemical substance on that particular path and thus the level keeps increasing.
Architecture:

Here, in the Architecture as shown in the figure, Due to the attenuation, there might be chances of losing connectivity or having signal losses. Also when there are more than one road side units, the iteration count is also taken into account to decide which RSU has to come into action. The iteration count keeps decreasing with respect to the time and it keeps increasing with the passage of more vehicles. So the working occurs in such a way that the message from a vehicle is passed onto a RSU and then from the RSU to another vehicle and so on. Thus as the iteration count increases, that particular RSU takes a upper hand in the communication network and also with this methods, the signal fading can also be reduces based on the sub-networks chosen to have the communication stable .

Also, such networks can be used in future vehicles to prevent further accidents and such by implementing this technique on to every vehicular device. But in this type of technique, the problems which occurs is that when in the time of accidents, there is no information storage which is occuring and hence, here there is just prevention, but no acknowledgement.

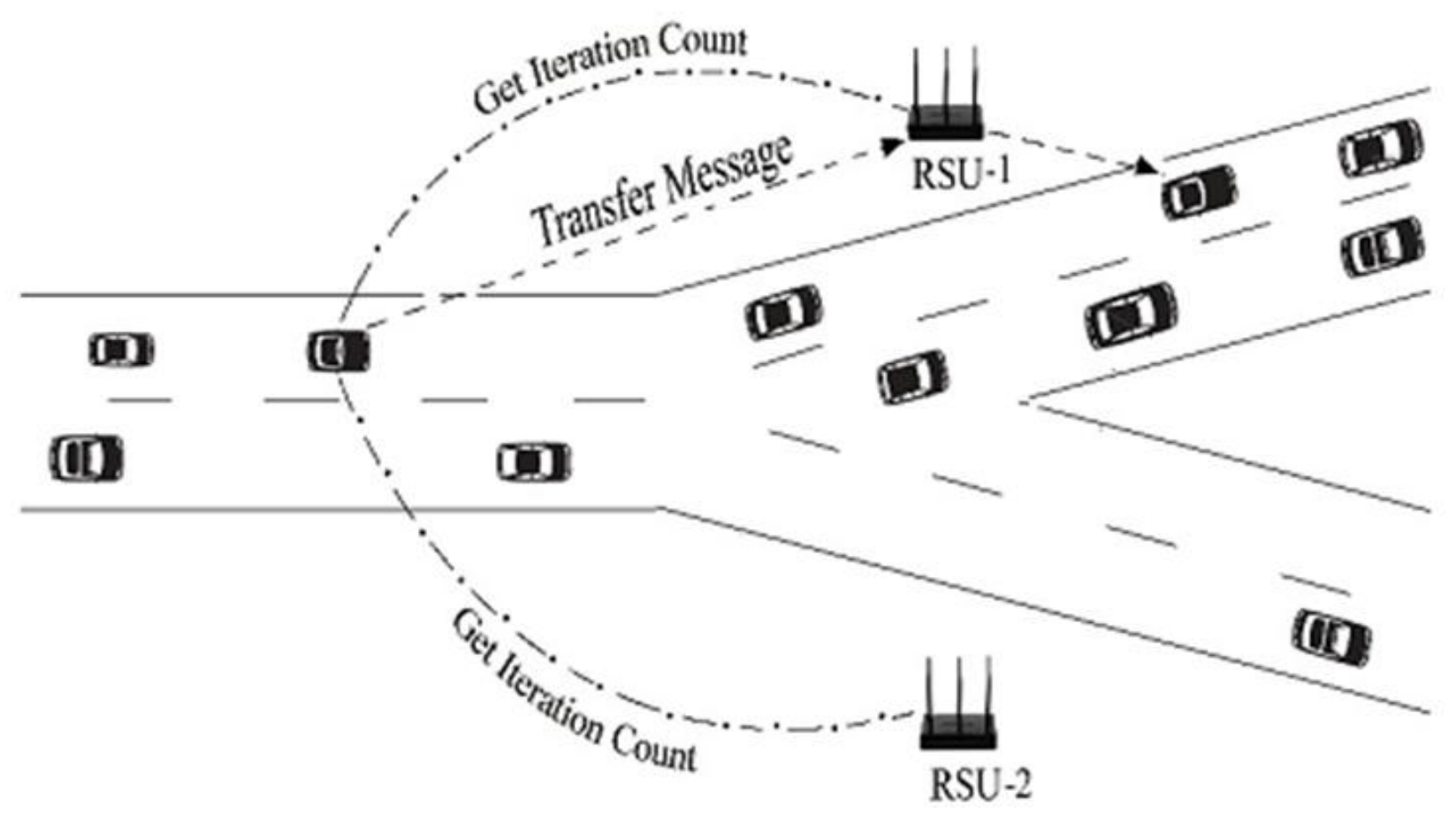

Fig 1 : Communication Network Using RSU in RACO.

\section{B. RF Technology}

The microcontroller is interfaced with the traffic light which are given as input at the traffic signal only. The light is given to the microcontroller directly. The microcontroller transfers the signals to RF Xbee transmitter through serial communication using UART. Now the transmitter sends the given signals to RF Xbee receiver which is fitted to the vehicles.
Automatic vehicle speed reduction using RF technology which reduces the speed of the vehicle by reducing the fuel rate of the vehicle where the vehicle automatically goes to the control and avoids the accidents .Transmitters are tuned at $433 \mathrm{MHZ}$ are mounted as these transmitters continuously radiate RF signal for some particular area. If vehicle comes within this radiation receiver fitted in the vehicle gets activated. 
In the RF Transmitter part, TWS -434 are very small and these are required for the applications of short range RF remote controls. The size of the trans module is one-third of the size of the postage stamp which can be placed easily inside a small plastic enclosure. The output of the trans is 8 $\mathrm{mW}$ at $433.92 \mathrm{Mhz}$ ranging 400 foot outwards. Indoor range is 200 foot approx. The TW is 434 trans allows both digital and linear inputs which operate from 1.5 to $12 \mathrm{~V}$ DC.

The Rfreq Receiver has the sensivity of 3UV and operates at 433.92 Mhz and the RW is 434 Receiver allows both didgital and linear outputs which are operating from 4.5 to 5.5 DC. RFreq antenna which is WC 418 , is made up of 26 gauge C-Steel which can be a simple Music wire which can be soldered to the PC boards. It is 6.8 inchs long and has a plastic coating for safety purpose which allows insertion of 1 inch in a PC board.

\section{HARDWARE DESIGN}

MicroController(ARM Based)

In order to generate a high speed flash memory of 128/ $512 \mathrm{Kbytes}$, the LPC2148 which is based on ARM based MicroController with the support of embedded trace and emulation in real-time. The unique accelerator architecture and 128bit wide memory interface allows a code execution which is 32 bit at max clock rate. For industrial control, medical system, access control and point of sale, the microcontrollers with compact 64pin package, various 32 bit timers, USB port low power consumption, 4 channels $10 \mathrm{bit}$ ADC, PWM ch and 46 GPIO are well suited.

\section{RF Xbee}

Among the most popular RF Xbee is a higher power version of it. This is good for P2P, Multipoint and it is possible to convert into mesh network point which is of 802.15.4 protocol with $60 \mathrm{~mW}$ wireless module. The Digi Xbee tool can configure Baud rate, power and sleep modes. In order to fit into a bread board, the pins on Xbee are of $2 * 10^{\wedge}(-3) \mathrm{m}$ spacing . The kit is having $250 \mathrm{mAmp}$ and $3.3 \mathrm{~V}$ regulators. It consists of wire antenna also.

\section{Mechanical setup}

The DC motor of $9 \mathrm{~V}$ battery is made used. Two DC motors are made use of to run the wheels at the rate of 500rpm and low torque is used to control the brakes having $10 \mathrm{rpm}$ speed.

\section{SOFTWARE DESIGN}

Flash magic

The flash based MController from NXP that is a PC tool for programming using a serial or Ethernet protocol while using it with hardware. In order to load the compiled program into the processorLPC2148 using serial communication cable. It supports half duplex communication, it is powerful and flexible in time code.

\section{RIDE}

Software tools such as compilers, assemblers and simulators are available in this kit. These are of Dynamic Link Library files ‘*.dll' and they are called from RIDE.

\section{Embedded C}

Atleast a single Main() function should be in a $\mathrm{C}$ program. In order to perform a function the instruction are combined together. These are treated as higher level operations.

\section{FUTURE SCOPE}

In the above section we have seen many techniques of reducing mishaps but still errors exist and hence we are stepping into a new technique where in a system is designed which will automatically control speed of vehicle in and around school, college and hospital areas and also instantaneous transfer of message is possible at the time of accidents.

In the current scenario we have CCTV cameras almost everywhere but still our department of police have trouble in identifying the incident. So this stands as a main issue and hence we go on to the below technique. So here, with the help of radio frequency transceivers automatic information is passed on to the nearest police station.

The transmitters which work on radio frequencies are fixed in various necessary locations which will identify the vehicles.The vehicle in turn are fitted with receivers which work on $\mathrm{R}$ frequencies which are capable of receiving the $\mathrm{R}$ freq signals from these locations, so the concept falls in such a way that when vehicle reaches the particular area the receiver receives the signal and passes on to the microcontroller.The microcontroller will detects this and reduces the speed or sound respectively, coming to the design part we will consider one IR transmitters and two receivers for the purpose of sensing the signals along with these features we can include another technique use which can detect pedestrians/obstacles. If the vehicle is in forward motion and if it reaches a pedestrian as an obstacle it immediately gives the warning signal to the one driving the vehicle.

The simultaneous transfer of message takes place by setting a threshold in the vibration and hence this way accidents can be acknowledged [6] After the incident is acknowledged and the simultaneous transfer is done a copy of the same is sent to the nearest police station and this is saved in there data base such that a person can access the data at any time.

In order to achieve the above mentioned technique the following hardware tolls are required. The ATMEL 89C51 can be used as Microcontroller because it has two advantages i.e. it has a flash memory and it is reprogrammable when compared to 8051.A 16*2 LCD display is used to display the alert signals. Software tolls which can be used are Embedded $\mathrm{C}$ and Kiel micro vision. 


\section{CONCLUSION}

The main issue faced in these current times is that lack of police reports on the road conditions of the occurred accidents. One of the way which can be used in solving this problem can be done by using $\mathrm{RF}$ (radio frequency) transceivers, such that there is a threshold vibration mode set and as the vehicles vibration mode exceeds this is automatically and instantly exchanged with the other vehicle which it hits, and also this information is passed on to the nearest police stations through the GSM technology. Security issues, routing issues and privacy issues etc are the challenges stated above that can be eliminated by using the efficient technique known as VANET, hence for safer and easy transportation this system can be effectively used. The outcome of our project results in minimize of traffic problems such as crashing of vehicles, collision aware and local danger alert. The RF technology is used to control the speed of the vehicles automatically. The input is the yellow light from the traffic signal which is given to the controller and RF Xbee modules are sent. The RF module does not require physical braking system due to this the cost of car is reduced. Now the signal is transmitted and received at another RfXbee which is placed at the vehicle. These received signals control the speed of the wheels and stops at the red light. This also has the advantages like reduction of speed, accident prevention, audio voice for blind people .The parameters that are used in this system are less expensive.

\section{REFERENCES}

[1] Jaya Sehgal, Poonam Arora, "Delay Optimization in VANET Using Ant Colony Optimization and WIMAX”,International journal of advanced research in electrical, Electronics and Instrumentation Engineering, August 2014. . (references)

[2] Debasmita Mukherjee, Sriyankar Acharyya,"Ant colony optimization technique applied in network routing problem", International journal of computer applications Volume 1 No 15, March 2008

[3] Gupta, D.K.; Arora, Y.; Singh, U.K.; Gupta, J.P.,"Recursive Ant Colony Optimization for estimation parameters of a function", Recent Advances in InformationTechnology (RAIT), 2012 1st International Conference on , vol., no., pp.448,454, 15-17 March 2012.

[4] Rezwana Karim, "VANET: Superior system for content distribution in vehicular network applications",AIRCC Publishing Corporation, January 2009.

[5] MHS Khandre, PS Kulkarni,"A Recursive Ant Colony System For Feature Extraction \& Edge Detection",2013,“ International Journal of Engineering” Element symbols.

[6] Saif Al-Sulthan, Ali H Al-Bayatti ,"Context aware driver behavior detection system in intelligent transport system",IEEE vol 15,2014.

[7] Wei Xiong school of automatic science nad technology china,"Vehicle node localizatio with GPS in VANET", International confernce on connecting vehicles and expo[ICCVE] pp no.1068-1071 2014

\section{BIOGRAPHIES}

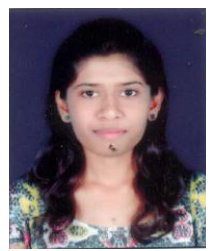

Arpithanath. $M$

$8^{\text {th }}$, sem, TCE Department.

Good in networking and routing, embedded system.

arpithanathm@gmail.com

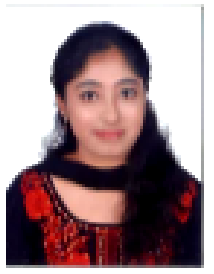

Suraiya Khanum A

$8^{\text {th }}$ sem, TCE Department.

Interested in $\mathrm{CCN}$, Networking and Security Analysis

suraiyakhanum294@gmail.com

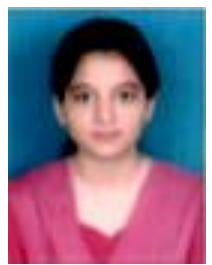

\section{Umaima Rafi}

$8^{\text {th }}$ sem, TCE Department.

Have knowledge in windows and computer applications.

umaima.rafi@gmail.com

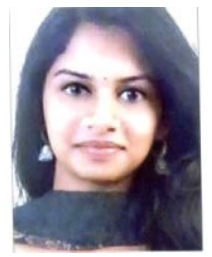

Vinaya Ananthi. $R$

$8^{\text {th }}$ sem, TCE Department.

Have adequate knowledge in embedded system, Microcontrollers, Networking and routing.

vinaya.ananthi@gmail.com

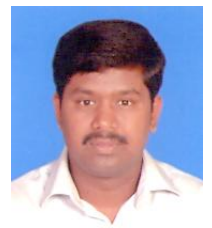

Under the guidance of Mr.Anand Swamy A $\mathbf{S}$ (Assistant Professor of ECE/TCE Department)

Working in ECE/TCE TJIT. Research Scholar in image processing domain anandswamyas@johngroup.com 